\title{
SCALING OF BUBBLING FLUIDIZED BED REACTORS WITH GLICKSMAN'S VISCOUS LIMIT SET AND CFD SIMULATION
}

\author{
R. K. THAPA \& B. M. HALVORSEN \\ Institute for Process, Energy and Environmental Technology, Telemark University College, Norway.
}

\begin{abstract}
Glicksman's viscous limit set of dimensionless parameters have been investigated using experimentally verified computational fluid dynamics model. Simulations have been performed for the two bubbling fluidized beds with different particle sizes and densities. Dimensionless average pressure drops across the bed height, dimensionless pressure standard deviations and dimensionless relative pressures have been investigated as a function of dimensionless superficial gas velocities for the two beds. Fluctuation of solid volume fraction and contours of solid volume fraction have also been investigated at different dimensionless gas velocities. Time series data of the pressure fluctuation and solid volume fraction are compared. The results indicate that the fluid dynamic similarity between two beds holds up to particle Reynolds number of 15 . After this, the bubble activities in the two beds start to deviate significantly. The results of the work show that the analysis of solid volume fraction fluctuation gives higher accuracy than time-series pressure fluctuation when scaling the bubbling fluidized bed within the viscous limit. Keywords: Fluidized bed, scaling, viscous limit, Glicksman, CFD.
\end{abstract}

\section{INTRODUCTION}

Scaling of fluidized bed reactors in a proper way remains a major challenge in process industries. Scaling of fluidized beds is still an inexact science rather than a mix of mathematics, witchcraft, history and common sense as indicated by Matsen [1]. The scaling law for fluidized bed reactors has been developed by Glicksman. The law is derived by non-dimensionalizing the governing fluid dynamic equations for gas-solid flow [2]. This gives a set of dimensionless parameters. For two fluidized bed reactors to be fluid dynamically similar, the set of dimensionless parameters should be matched. The set of the dimensionless parameters is used to developed lab-scale cold models that simulate the flow behavior of an operating plant. This enables to improve the flow behavior of an existing plant when it is required. In addition, scaling is useful for the modification of the existing plants.

Glicksman has derived two sets of dimensionless parameter for scaling of fluidized beds: full set and simplified set. In the full set, dimensionless parameters such as Froude number, Reynolds number, density ratio, bed to particle size ratio, bed geometry ratio, particle sphericity and particle size distribution should be matched. van Ommen et al. studied the simplified set, full set and extended full set with additional dimensionless pressure group and found reasonable agreements [3]. In the particular application such as biomass gasification reactors, it is difficult to match all the parameters of the set. Sometimes exotic particles (very high density and very low particle size) are required when it comes to scaling down a very large operating plant to a lab-scale cold model [4]. To overcome this problem, Glicksman has simplified the full set. In the simplified set, Reynolds number has been replaced by the ratio of excess gas velocity to minimum fluidization velocity and the ratio of particle diameter to bed diameter has been omitted [5]. The simplified set gives more flexibility in selecting dimensions for the small-scale reactors. 
The simplified set is divided into two flow conditions. When the fluid-particle drag is dominated by inertial forces, it is the inertial limit. When the drag is dominated by viscous forces, it is the viscous limit. This division allows to consider the viscous dominated and inertial dominated flow separately [6]. In the viscous limit, the gas-particle density ratio is omitted for lower particle Reynolds numbers. For the particles with particle Reynolds number up to 4, the particle to gas density ratio can be omitted in the dimensionless set of parameters. This makes the viscous limit set of dimensionless parameters more flexible for scaling of fluidized bed. Bubbling fluidized bed reactors operate comparatively at lower gas velocities. Geldart A and B particles with lower gas velocities have low particle Reynolds numbers. Therefore, the viscous limit set is particularly suitable for scaling of bubbling fluidized bed reactors. In the literature, some authors have claimed that the viscous limit of the particle Reynolds numbers is higher than that given by Glicksman. The range of particle Reynolds numbers is not consistent. Stein et al. [7] have claimed that the density ratio can be neglected up to particle Reynolds number 100. Farrel [8] has shown that the viscous limit set does not hold for the particle Reynolds number between 10 and 25. Various reasons are responsible for the deviations.

The scaling of bubbling fluidized bed should be guided by the well understanding of particle properties and flow regimes in the bed. The scaling laws are derived from the set of independent dimensionless parameter. The dependent variables such as pressure and void fraction should also be compared in their non-dimensional form.

Downscaling of a large reactor to small lab-scale model sometimes requires significant reduction of particle size to maintain the dimensionless parameters. Particles with very different particle sizes belong to different Geldart groups. Different Geldart group particles have different flow regimes and this may result in different bubble behaviors in the scaled bed [9]. The selection of particle size and densities for scaled bed should be within the same Geldart group of particles. Scaling from very large commercial scale to small lab-scale cold model may also change the operating velocities between the two beds significantly. This may result in changing flow regime and consequently the bubble behavior of the two beds. In addition to this, the bed geometry also needs scaling. In practice, experimental investigation of all these conditions is not possible.

Computational fluid dynamic (CFD) models are valuable tools for this situation. The CFD tools have a significant potential in predicting the effect of scaling in hydrodynamics of fluidized beds [10]. Any particle density and size can be selected for the simulation of the model. The result of the simulations can serve as proper guidelines on selection of particle size, density and scaled bed geometry. This helps to avoid many intermediate errors in constructing lab-scaled cold model of an operating plant.

\section{VISCOUS LIMIT SET OF SCALING RULES}

The viscous limit set is derived by Glicksman for dense fluidized bed with low gas velocities [5]. In the dense region of the bed, the viscous forces are dominant compared with the inertial forces. Due to insignificant effect of inertial forces, requirements of dimensionless parameters to be matched are less. Equation (1) shows the viscous limit set of dimensionless parameters. Application of the set requires similarity of Froude number, the ratio of excess gas velocity to minimum fluidization velocity, bed geometry ratio, particle sphericity and particle size distribution. In addition to this, the particle Reynolds number should not exceed the value of 4 . This criterion is shown in eqn (2). In practice, the viscous limit set of dimensionless parameters can 
be applied for scaling of bubbling fluidized beds. The particles of the large bed as well as scaled bed should have lower particle size.

$$
\begin{gathered}
\frac{u_{0}^{2}}{g L}, \frac{u_{0}}{u_{m f}}, \frac{L_{1}}{L_{2}}, \phi, \text { particle size distribution } \\
\operatorname{Re}=\frac{\rho_{g} u_{0} d_{p}}{\mu}<4
\end{gathered}
$$

\section{COMPUTATIONAL MODELS}

Two approaches in modeling of gas-solid multiphase flow have been applied currently: Eulerian-Lagrangian and Eulerian-Eulerian. Eulerian-Lagrangian model tracks the motion of each particle and solves the dynamics of the fluid at the length scale smaller than the particle diameter. The modeling approach requires large computer memory and time for simulation. Eulerian-Eulerian model treats both the fluid and solid phases as interpenetrating continua and solves the dynamics averaging their equation of motion. The modeling approach is computationally less demanding [11]. The Eulerian-Eulerian approach is implemented in modeling the fluid dynamics of fluidized bed. To treat the particles as continuum, kinetic theory of granular flow is applied. The kinetic theory of granular flow considers the conservation of solid fluctuation energy [12]. The simulations have been performed using commercial CFD software ANSYS/Fluent 13.0.

The CFD model used in this work was validated against the experimental data. The experimental measurements were performed in a cold model with pressure sensors. Experimental data such as pressure drop across the bed height, pressure standard deviation and bubble behavior were compared with computational prediction of the model. A good agreement between the experimental measurements and the model predictions were established. The details of the validation work performed by the authors can be found in WIT Transactions on Engineering Series 2012 [13].

Two-dimensional simulations have been performed with air as fluidizing gas. The coefficient of restitution is set to 0.9 . The coefficient represents the elasticity of collision between the particle-particle and particle-wall. Variation in the coefficient of restitution affects on fluid dynamic similarities between two beds $[14,15]$. The simulation is initialized with solid volume fraction of 0.6. In the simulations, the particles in both beds are assumed as spherical monosized particles. The simulation time for reference and scaled bed are 9.2 and $8 \mathrm{~s}$, respectively. The simulation time is different for two beds to adjust the scaling factor with time.

The locations of the surface monitors for the two beds are presented in Fig. 1. In each bed, 25 surface monitors are set to record the pressure fluctuations and solid volume fraction fluctuations. The monitors are located at $0 \%, 25 \%, 75 \%$ and $100 \%$ along both the bed height and the bed width. In addition to this, line monitors are set at the bed height of $25 \%, 50 \%$ and $75 \%$ for the solid volume fraction fluctuation.

The properties of particles and gases used in the model for the two beds are presented in Table 1.

To start the simulation, theoretical minimum fluidization velocities for the particles are calculated using the following equation:

$$
\frac{\left(\phi \cdot d_{p}\right)^{2}\left(\rho_{p}-\rho_{g}\right) \cdot g}{150 \mu_{g}} \cdot \frac{\varepsilon_{m f}^{3}}{1-\varepsilon_{m f}}
$$




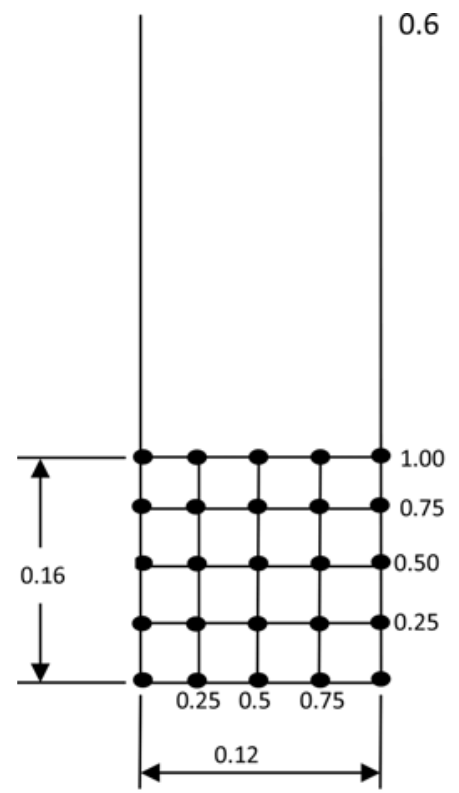

(a)

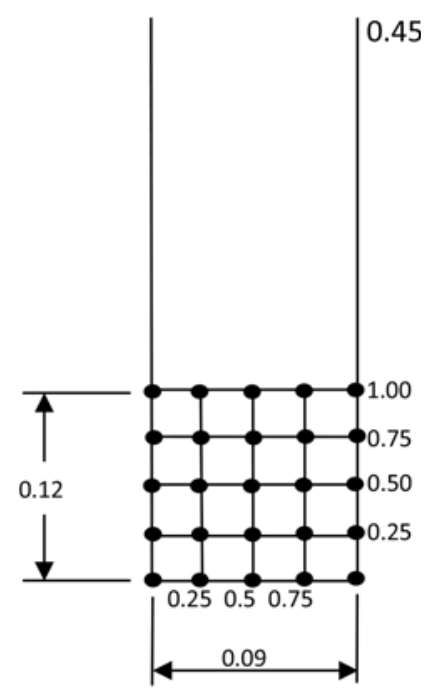

(b)

Figure 1: Bed dimensions and location of surface monitors: (a) reference bed and (b) scaled bed.

Table 1: Properties of particles and gases.

\begin{tabular}{lcccccccc}
\hline Set & $\begin{array}{c}d_{p} \\
(\mu \mathrm{m})\end{array}$ & $\begin{array}{c}\rho_{p} \\
\left(\mathrm{~kg} / \mathrm{m}^{3}\right)\end{array}$ & $\begin{array}{c}\rho_{g} \\
\left(\mathrm{~kg} / \mathrm{m}^{3}\right)\end{array}$ & $\begin{array}{c}\mu_{g} \\
(\mathrm{~Pa} \cdot \mathrm{s})\end{array}$ & $\begin{array}{c}u_{m f} \\
(\mathrm{~m} / \mathrm{s})\end{array}$ & $\begin{array}{c}H \\
(\mathrm{~m})\end{array}$ & $\begin{array}{c}D \\
(\mathrm{~m})\end{array}$ & $\begin{array}{c}\text { Geldart } \\
\text { group }\end{array}$ \\
\hline Reference bed & 320 & 2500 & 1.225 & $1.78 \cdot 10^{-5}$ & 0.147 & 0.16 & 0.12 & $\mathrm{~B}$ \\
Scaled bed & 240 & 3800 & 1.225 & $1.78 \cdot 10^{-5}$ & 0.170 & 0.12 & 0.09 & $\mathrm{~B}$ \\
\hline
\end{tabular}

The gas velocities and the dimensionless parameters of the two beds used in the simulation are presented in Table 2.

\section{RESULTS AND DISCUSSION}

Pressure fluctuation is most commonly used in the studies of fluidized beds. In bubbling fluidized bed, the pressure fluctuation is related to the bubble motion in the bed. A single pressure point contains the global information about the bed. Many researchers have attempted to validate fluid dynamic similarity between the bubbling fluidized beds from time-series pressure data [16]. The standard deviation of pressure fluctuation is an indirect method to identify the fluid dynamics in fluidized beds [17].

The pressure fluctuations in the bed are monitored at the equally distributed 25 points within the bed. The dimensionless pressure standard deviation is the ratio of pressure standard deviation at identical points of the two beds with identical dimensionless gas velocity. The dimensionless gas velocity is the ratio of excess gas velocity to minimum fluidization velocities of the two beds. The dimensionless pressure standard deviation as a function of dimensionless gas velocity is presented in Fig. 2. For two beds to be fluid dynamically 
Table 2: Gas velocities and dimensionless parameters used in the simulation.

\begin{tabular}{llcccr}
\hline Simulation & \multicolumn{1}{c}{ Set } & $\begin{array}{c}u_{0} \\
(\mathrm{~m} / \mathrm{s})\end{array}$ & $\begin{array}{c}u_{0}^{2} / g d_{p} \\
(-)\end{array}$ & $\begin{array}{c}u_{0} / u_{m f} \\
(-)\end{array}$ & $\operatorname{Re}(-)$ \\
\hline \multirow{3}{*}{1} & Reference bed & 0.170 & 9.19 & 1 & 2.43 \\
\multirow{3}{*}{2} & Scaled bed & 0.147 & 9.24 & & 3.73 \\
& Reference bed & 0.340 & 36.75 & 2 & 4.86 \\
3 & Scaled bed & 0.294 & 36.98 & & 7.46 \\
\multirow{4}{*}{4} & Reference bed & 0.510 & 82.69 & 3 & 7.28 \\
& Scaled bed & 0.441 & 83.20 & & 11.20 \\
5 & Reference bed & 0.680 & 147.00 & \multirow{2}{*}{4} & 9.71 \\
& Scaled bed & 0.588 & 147.9 & & 14.93 \\
& Reference bed & 0.850 & 229.69 & \multirow{2}{*}{5} & 12.14 \\
& Scaled bed & 0.735 & 231.10 & & 18.66 \\
\hline
\end{tabular}

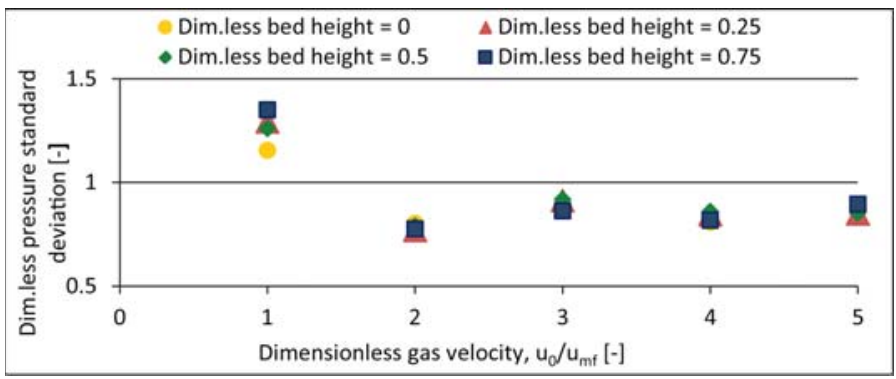

Figure 2: Dimensionless pressure standard deviation as a function of dimensionless gas velocity at different bed heights.

similar, the dimensionless pressure standard deviation should be similar for the same dimensionless gas velocity at any identical points of the beds. But the dimensionless pressure data need not to be equal to 1 . This requirement applies only to Glickman's full sets and inertial limit sets of dimensionless parameters. In the viscous limit set, the density ratio between two beds does not need to be matched. This makes the set more attractive for scaling of fluidized bed reactors within the limited particle Reynolds number. The figure shows pressure standard deviations of the two beds at $0 \%, 25 \%, 50 \%$ and $75 \%$ of the bed heights at five different dimensionless gas velocities. The dimensionless pressure standard deviations are almost same for those points of the bed at the dimensionless gas velocities of 2-5. This shows the fluid dynamic similarity of the beds at gas velocities higher than minimum fluidization. The dimensionless pressure standard deviation at the dimensionless gas velocity of 1 has a slight deviation. Particularly, the dimensionless pressure standard deviation at the bottom of the bed has different value than other points along the height of the bed. The dimensionless velocity of 1 represents the transition of the beds from static condition to fluidizing condition. Slight difference in initial minimum fluidization velocity set in the simulation may result in such types of deviations. However, the deviation is within the acceptable error limit. 
Table 3: Pressure standard deviation at dimensionless bed height of 0.5 .

\begin{tabular}{|c|c|c|c|}
\hline \multirow{2}{*}{$\begin{array}{l}\text { Dimensionless } \\
\text { gas velocity (-) }\end{array}$} & \multicolumn{2}{|c|}{ Pressure standard deviations $(\mathrm{Pa})$} & \multirow{2}{*}{$\begin{array}{l}\text { Dimensionless } \\
\text { pressure standard } \\
\text { deviation }(-)\end{array}$} \\
\hline & Reference bed & Scaled bed & \\
\hline 1 & 24.41 & 19.35 & 1.26 \\
\hline 2 & 151.45 & 191.91 & 0.78 \\
\hline 3 & 281.10 & 306.41 & 0.91 \\
\hline 4 & 338.68 & 393.75 & 0.86 \\
\hline 5 & 405.35 & 475.14 & 0.85 \\
\hline
\end{tabular}

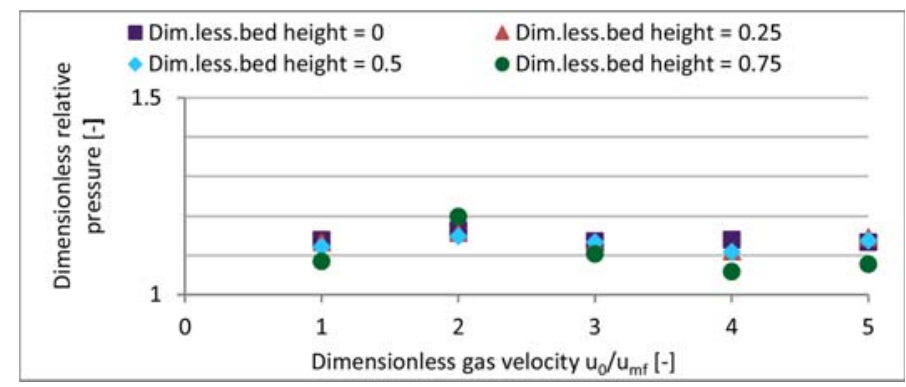

Figure 3: Dimensionless relative pressure as a function of dimensionless gas velocity at different bed heights.

The pressure standard deviation increases with gas velocity. The pressure standard deviations for both the beds taken separately have minimum values at the minimum fluidization velocity. The pressure standard deviation at the dimensionless bed height of 0.5 is presented in Table 3.

Dimensionless relative pressure as a function of dimensionless gas velocity is presented in Fig. 3. The dimensionless relative pressure is the ratio of the pressure along the same dimensionless bed height.

Dimensionless relative pressure does not show significant difference along the bed height with increasing dimensionless gas velocities. However, the dimensionless relative pressure is slightly decreased at dimensionless gas velocities of 4 and 5. This indicates the deviation of relative pressure at these dimensionless gas velocities in comparison to the others.

The dimensionless average pressure drop as a function of dimensionless gas velocity is shown in Fig. 4. The average pressure drop is the ratio of pressure drop across the bed height to the total height of the bed. In the viscous limit set, the density ratio of the particles is not maintained. The differences in the particle to gas density ratio result in different average pressure drop across the bed height. Therefore, the average pressure drop across the bed can only be compared in its dimensionless numbers. As indicated in the figure, the dimensionless values of average pressure drop are increased more at the dimensionless gas velocities of 4 and 5 .

The increased dimensionless average pressure drop at dimensionless gas velocities 4 and 5 indicates the deviation of pressure fluctuation in the two beds for these dimensionless gas 


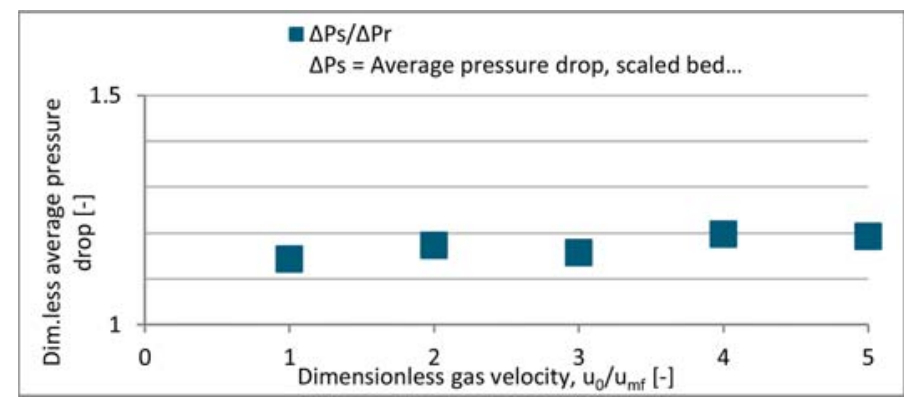

Figure 4: Dimensionless average pressure drop across the bed height as a function of dimensionless gas velocity.

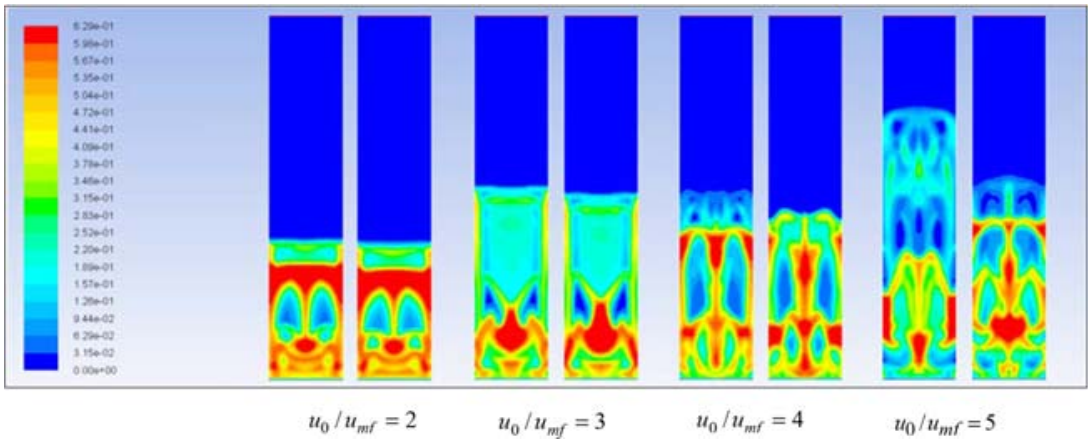

Figure 5: Contours of solid volume fraction at different dimensionless gas velocities at 9.2 and $8 \mathrm{~s}$ of simulation time for reference and scaled bed, respectively. Left: reference bed and right: scaled bed.

velocities. The deviation is significant as the particle Reynolds number numbers approaches about 15 and 19, respectively. The contours of solid volume fraction at these dimensionless gas velocities shown in Fig. 5 also confirm the significant deviation, suggesting the viscous limit set is valid up to the particle Reynolds number $<15$.

Analysis of time series of pressure fluctuation data in the two beds shows that the pressure fluctuations in the beds do not show significant deviation even the particle Reynolds number is significantly higher than the viscous limit. However, at the dimensionless gas velocities of 4 and 5, slight deviation of dimensionless pressure standard deviation, dimensionless relative pressure and dimensionless average pressure drop can be seen from the figures. It shows that the fluctuation of pressure between two beds at these velocities deviate significantly.

Detailed information about the bubble activities in the bed can be obtained from the data of solid volume fraction fluctuation. The fluctuation of solid volume fraction in a bubbling fluidized bed is due to the bubble activities. The contours of solid volume fraction for the two beds are presented in Fig. 5. The contours are presented from dimensionless gas velocity 2-5. The contours are snapshots taken at the simulation time of 9.2 and $8 \mathrm{~s}$ for reference and scaled bed, respectively. The simulation time is different because of the scaling factor. The ratio between the scaling times is 1.15 , which is the scaling factor of the beds. As long as the 


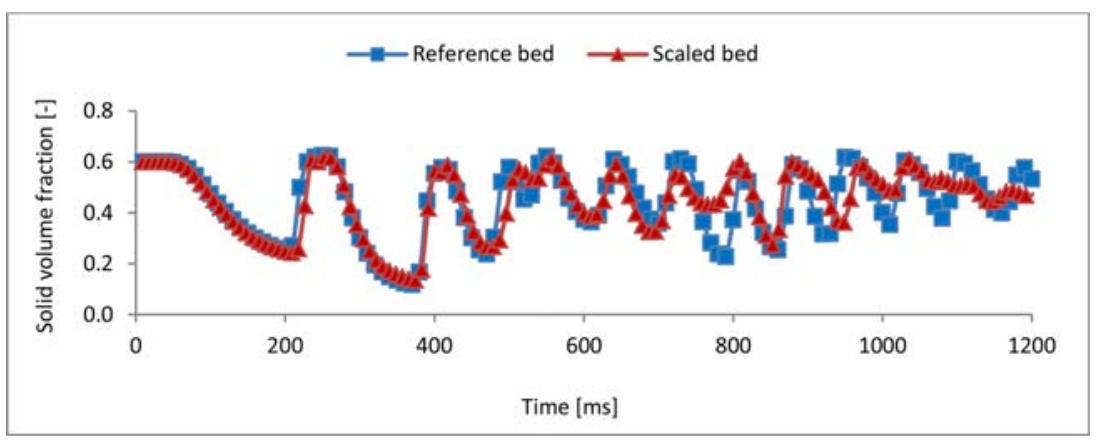

Figure 6: Solid volume fraction fluctuation with time. Dimensionless bed height $=0.5$, dimensionless bed width $=0.75$ and dimensionless gas velocity $=2$.

time is also scaled, the solid volume fraction of the beds should be similar at a given time for two beds to have fluid dynamic similarity.

At the dimensionless gas velocity of 1 , the beds are static and there are no bubbles in the bed. The contours of solid volume fraction for two beds at the dimensionless gas velocity of 2 and 3 are similar. This shows that the bubble formation and bubble activities in the bed are similar. The contours show negligible deviation between the two beds. The highest particle Reynolds number at these velocities is about 11. Up to this value of particle Reynolds number, the bubble behavior of the bed is similar. At the dimensionless gas velocity of 4 , the contours show some deviation between the bubble activities in the beds. The particle Reynolds number for the dimensionless gas velocity is about 15 . This is the Reynolds number at which the deviation of the bubble activities between the two beds starts. At the dimensionless gas velocity of 5 , the contours deviate significantly. The particle Reynolds number at this velocity is about 18 .

The time-series data of solid volume fraction fluctuations for the two beds are presented in Fig. 6. The time series of solid volume fraction fluctuation in the beds gives more precise information of bubble activities. The figure shows the solid volume fraction fluctuation at a single point. Similarity in the fluctuation of solid volume fraction between the two beds means the similar bubble behavior in the beds.

\section{CONCLUSION}

Glicksman's viscous limit set of dimensionless parameters are applied in scaling of bubbling fluidized beds with different particle sizes and densities. The beds are simulated using experimentally validated CFD model. The simulations are performed in commercial CFD software ANSYS/Fluent 13.0. Dimensionless pressure standard deviation, relative pressure and average pressure drop across the bed height are investigated as a function of dimensionless superficial gas velocities. Dimensionless pressure standard deviation is similar for the two beds at the dimensionless gas velocities $2-5$. The dimensionless relative pressure and dimensionless average pressure drop deviate at dimensionless gas velocities of 4 and 5 .

Fluctuation of solid volume fraction and contours are investigated as a function of dimensionless gas velocity. The contour of solid volume fraction is similar for the two beds at the dimensionless gas velocities of 2 and 3 . The contour starts to deviate from the dimensionless gas velocity of 4 and deviates significantly at the velocity 5 . The similarity of solid volume fraction fluctuation or the bubble activities in the bed holds for the particle Reynolds number up to 15 . 
CFD computational fluid dynamics

\section{NOMENCLATURE}

$D \quad$ bed diameter $(\mathrm{m})$

$d p \quad$ particle diameter $(\mathrm{m})$

$g \quad$ acceleration due to gravity $\left(\mathrm{m} / \mathrm{s}^{2}\right)$

$H \quad$ static height of bed (m)

$L_{1}, L_{2} \quad$ bed geometry (m)

Re Reynolds number $(-)$

$u_{0} \quad$ excess gas velocity $(\mathrm{m} / \mathrm{s})$

$u_{m f} \quad$ minimum fluidization velocity $(\mathrm{m} / \mathrm{s})$

$\rho_{p} \quad$ particle density $\left(\mathrm{kg} / \mathrm{m}^{3}\right)$

$\rho_{g} \quad$ gas density $\left(\mathrm{kg} / \mathrm{m}^{3}\right)$

$\mu_{g} \quad$ gas viscosity $(\mathrm{Pa} \cdot \mathrm{s})$

$\phi \quad$ particle sphericity (-)

$\varepsilon_{m f} \quad$ void fraction at minimum fluidization (-)

\section{REFERENCES}

[1] Matsen, J.M., Scale-up of fluidized bed processes: principle and practice. Powder Technology, 88(3), pp. 237-244, 1996. doi: http://dx.doi.org/10.1016/S00325910(96)03126-9

[2] Glicksman, L.R., Scaling relationships for fluidized beds. Chemical Engineering Science, 39(9), pp. 1373-1379, 1984. doi: http://dx.doi.org/10.1016/0009-2509(84)80070$\underline{6}$

[3] van Ommen, J.R., et al., Computational validation of the scaling rules for fluidized bed. Powder Technology, 163, pp. 32-40, 2006. doi: http://dx.doi.org/10.1016/j.powtec.2006.01.010

[4] Rüdisüli, M., et al., Scale-up of bubbling fluidized bed reactors - a review. Powder Technology, 217(0), pp. 21-38, 2012. doi: http://dx.doi.org/10.1016/j.powtec.2011.10.004

[5] Glicksman, L.R., Hyre, M. \& Woloshun, K., Simplified scaling relationships for fluidized beds. Powder Technology, 77(2), pp. 177-199, 1993. doi: http://dx.doi. org/10.1016/0032-5910(93)80055-F

[6] Sanderson, J. \& Rhodes, M.J., Hydrodynamic similarity of solids motion and mixing in bubbling fluidized beds. AIChE Journal, 49(9), pp. 2317-2327, 2003. doi: http://dx.doi. org/10.1002/aic.690490908

[7] Stein, M., Ding, Y.L. \& Seville, J.P.K., Experimental verification of the scaling relationships for bubbling gas-fluidised beds using the PEPT technique. Chemical Engineering Science, 57(17), pp. 3649-3658, 2002. doi: http://dx.doi.org/10.1016/S00092509(02)00264-6

[8] Farrel, P.A., Hydrodynamic Scaling and Solid Mixing in Pressurized Bubbling Fluidized Bed Combustors, Massachusetts Institute of Technology: Massachusetts, 1996.

[9] Kunnii, D. \& Levenspiel, O., Fluidization Engineering. 2nd edn., Butterworth-Heinemann: USA, 1991.

[10] Knowlton, T.M., Karri, S.B.R. \& Issangya, A., Scale-up of fluidized-bed hydrodynamics. Powder Technology, 150(2), pp. 72-77, 2005. doi: http://dx.doi.org/10.1016/j.powtec.2004.11.036

[11] Mazzei, L., Eulerian Modelling and Computational Fluid Dynamics Simulation of Mono and Polydisperse Fluidized Suspensions, University College London: UK, 2008. 
144 R. K. Thapa \& B. M. Halvorsen, Int. J. Comp. Meth. and Exp. Meas., Vol. 2, No. 2 (2014)

[12] Gidaspow, D., Multiphase Flow and Fluidization, Academic Press Inc.: California, 1994.

[13] Thapa, R.K. \& Halvorsen, B.M., Validation of CFD model for prediction of flow behaviour in fluidized bed reactors. WIT Transactions on Engineering Sciences, Vol. 74, WIT Press: Southampton, pp. 231-239, 2012.

[14] Detamore, M.S., et al., A kinetic theory analysis of the scale-up of circulating fluidized beds. Powder Technology, 115, pp. 190-203, 2001. doi: http://dx.doi.org/10.1016/ S0032-5910(00)00397-1

[15] Didwania, A.K. \& Catolia, R.J., CFD simulation scale-up of a dual fluidized bed gasifier for biomass. Seventh International Conference on CFD in the Minarals and Process Industries, Melbourne, Australlia, 2009.

[16] Brown, R.C. \& Brue, E., Resolving dynamical features of fluidized beds from pressure fluctuations. Powder Technology, 119(2-3), pp. 68-80, 2001. doi: http://dx.doi. org/10.1016/S0032-5910(00)00419-8

[17] van Ommen, J.R., et al., Time-series analysis of pressure fluctuations in gas-solid fluidized beds - a review. International Journal of Multiphase Flow, 37(5), pp. 403-428, 2011. doi: http://dx.doi.org/10.1016/j.ijmultiphaseflow.2010.12.007 